\title{
Controlling helicity-correlated beam asymmetries in a polarized electron source
}

\author{
K.D. Paschke \\ University of Virginia, VA, USA
}

\begin{abstract}
The control of helicity-correlated changes in the electron beam is a critical issue for the next generation of parity-violating electron scattering measurements. The underlying causes and methods for controlling these changes are reviewed with reference to recent operational experience at Jefferson Lab.
\end{abstract}

\section{Introduction}

High-flux measurements in the next generation of parity-violating electron-scattering (PVES) experiments planned at Jefferson Lab aim to achieve a precision on the parityviolating asymmetry $\left(A_{P V}\right)$ in the range of 5-10 parts per billion $(\mathrm{ppb})$. These goals represent an order-of-magnitude improvement in statistical uncertainty compared to the recently reported HAPPEX-II measurement of APV from hydrogen, with a precision of $\approx$ $100 \mathrm{ppb}$. Systematic uncertainties must also be reduced by a similar factor. Without further improvement, a leading source of systematic uncertainty in these experiments may be helicity-correlated beam asymmetries (HCBA).

The term HCBA generically refers to any difference in the beam between the right- and left-handed $(R$ and $L)$ longitudinal-polarization states. An asymmetry in the total charge delivered to the target for each helicity state (the charge asymmetry $\mathrm{A}_{Q}$ ) will result in a detected asymmetry in the scattered flux. The correction for $\mathrm{A}_{Q}$ is generally limited to $\sim 1 \%$ by the accuracy of the measurement of $\mathrm{A}_{Q}$ or some non-linearity in the experimental apparatus. Future experiments will seek to hold $\mathrm{A}_{Q}$ to the level of $0.1 \mathrm{ppm}$; in the absence of any effort to control it, $A_{Q}$ would typically be larger by four orders of magnitude.

Because the differential cross-section is a sensitive function of energy and angle, any helicity-correlated change in average position, angle, or energy of the beam, is reflected in the detected scattering rate asymmetry. The accuracy of corrections for beam position differences are typically limited to $\sim 10 \%$ due to uncertainties in the measurement of the sensitivity of detected rates to the beam parameters.

The effect of position differences is suppressed by the symmetric acceptance of the spectrometers used for these measurements. However, this symmetry does not suppress sensitivity to spot size changes, or other beam shape changes. While effects due to beam spot size or shape changes have been assumed to be negligible in most recent experiments, simulations have suggested that such effects may be important in planned future experiments.

Asymmetries in the time domain may also be significant due to non-linearity of the experimental apparatus. One such example would be a significant non-linearity in position sensitivity. At Jefferson Lab, a strong time dependence of the HCBA has been observed at the few millisecond time scale, even when the average HCBA was small when integrated over the full 33.3ms helicity windows. Although the net effect was negligible for the HAPPEX-II measurements, it may prove to be important for future measurement goals. The underlying mechanisms for $A_{Q}$ and position differences will also 
produce spot size, shape, and time domain differences. Previous publications have detailed many of these mechanisms [1-3]; here the topic is reviewed and related to recent operational experience at Jefferson Lab.

\section{I dentifying the causes of HCBA}

\subsection{The basic features of the electron source}

The polarized electron source used for PVES measurements is based on the use of a doped GaAs photocathode. The dopant induces a strain in the photocathode crystal which breaks a degeneracy in a valance band, allowing spin-selective promotion of electrons to the conduction band using circularly polarized laser light. Once in the conduction band, a negative work-function from the cesiated surface allows these polarized electrons to exit the cathode and be accelerated in an electrostatic field. The laser light polarization state is prepared using a Pockels cell, an electro-optic device in which the birefringence is proportional to the applied electric field. A schematic of the optics used to prepare the laser light is shown in fig. 1.

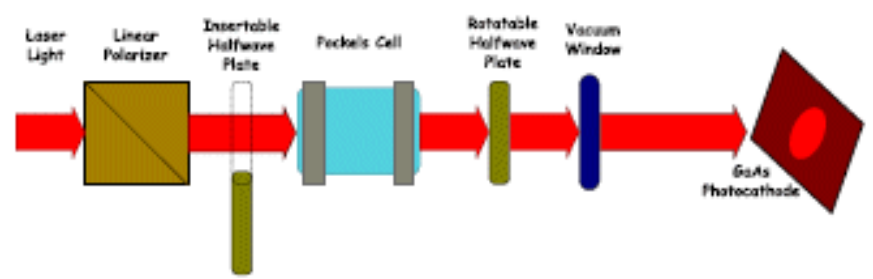

Fig. 1. Schematic of the optics used to prepare the circularly polarized laser light in the electron source.

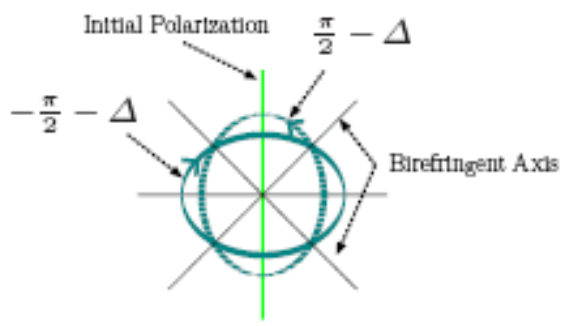

Fig. 2. Polarization ellipse, representing the shape circumscribed by the electric field vector, for each polarization state. An asymmetric phase shift $\Delta$ creates a small residual linear polarization of opposite sign for the two helicity states.

The laser light is initially linearly polarized in the vertical direction. The optic axis of the Pockels cell is aligned along the beam, with the birefringent axes at $\pm 45^{\circ}$ to this initialstate polarization. High voltage is applied to the Pockels cell appropriate to create a $\pm \Pi / 2$ phase-shift between the birefringent axes, thus creating left- or right-handed circular polarization. The beam then enters a high-vacuum system through a transparent window and illuminates the GaAs photocathode. The two half-wave plates shown in fig. 1 are not essential for the production of circularly polarized light; their function in controlling HCBA will be described below. 


\subsection{Effect of imperfect circular polarization}

The most troublesome source of HCBA is the imperfect circular polarization of the laser light. Following the prescription from [1], it is convenient to write the applied phase shift from the Pockels cell as:

$$
\delta^{\mathrm{R}}=-\left(\frac{\pi}{2}+\alpha\right)-\Delta ; \quad \delta^{\mathrm{L}}=+\left(\frac{\pi}{2}+\alpha\right)-\Delta,
$$

where $\mathrm{R}$ and $\mathrm{L}$ represent the sign of the produced circular polarization, and $\mathrm{a}$ and $\Delta$ parameterize the difference between the applied phase shift and a perfect $n / 2$ phase shift. Non-zero a implies that both states are symmetrically under or over phase-shifted, so that both the $R$ and $L$ states have a residual linear polarization with the same sign. Non-zero _ implies that the magnitude of the phase shift is different between the $L$ and the R states so that one is over-shifted and the other under-shifted, resulting in the two states possessing oppositely signed residual linear-polarization, as illustrated in fig. 2.

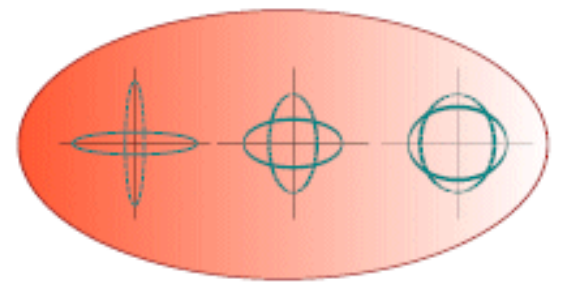

Fig. 3. Illustration of the effect of a phase gradient across a beam spot. A large $A_{Q}$ will be produced on the left side of the beam spot due to the higher degree of linear polarization, leading to a helicity-correlated shift in the beam centroid.

The strain on the photocathode, which is critical for producing the highly polarized electron beam, induces an analyzing power for linear polarization; one linear polarization state produces more current than an equal intensity of the opposite linear polarization. For historical reasons, the interaction of linear polarization with the analyzing power of the photocathode is referred to as the "PITA" effect [1]. The PITA effect, in various forms and from various sources, is responsible for most (but not all) of HCBA.

It should be noted that the $\Delta$ phase-shift contributes with the same sign to each helicity state, that is to say, it is a static effect uncorrelated to the voltage applied to the Pockels cell. Thus any static birefringent optics element contributes to this $\Delta$ phase shift. The Pockels cell itself possesses residual birefringence, due to latent stress on the crystal. The vacuum window also displays stress-induced birefringence, which introduces an additional source of $\Delta$ phase-shift, at an arbitrary axis with respect to the Pockels cell axes.

\subsection{Phase gradients}

Section 2.2 describes how a charge asymmetry is created by a non-zero $\Delta$ phase-shift. In general, the source of $\Delta$ is not uniform, which implies that $\mathrm{A}_{Q}$ can vary across the beam spot. Figure 3 illustrates this effect: $A_{Q}$ is large at the left of the beam spot and small at the right of the spot, implying that the centroid of the charge distribution is shifted between helicity states. The shift in the beam centroid can be thought of as the 
first moment of the intensity asymmetry; higher-order moments are also possible. In particular, while a $\Delta$-phase gradient will lead to a position difference, the second derivative of the $\Delta$ - phase will create a spot size asymmetry.

While the above-argument applies to a non-uniform $\Delta$ - phase, a similar effect is seen from a non-uniform analyzing power at the photocathode. A gradient (or second derivative) of the analyzing power will combine with a non-zero $\Delta$ to create position and spot size differences. One clear difference between the two effects is that position differences due to an analyzing-power gradient are proportional to the average $\Delta$, while those from a $\Delta$-phase gradient are the same regardless of the average $\Delta$ phase-shift. This difference is a useful diagnostic tool for distinguishing the two effects.

\subsection{Role of beam divergence}

It has long been recognized that a well-aligned Pockels cell is key to producing highly circularly polarized laser light. If the direction of beam propagation is not aligned to the Pockels cell optical axis, an offset birefringence is introduced. In studies leading up to the installation of the HAPPEX-II experiment at Jefferson Lab, it was recognized that the divergence of the laser beam produces a coupling of position and phase-shift. Consider a simple ray-trace view of a diverging (far-field) beam. In a far-field beam spot, one can imagine diverging optic rays with a strong correlation between a position in the beam spot and the angle of the associated optic ray. Because the $\Delta$ phase-shift due to the Pockels cell varies with the angle of the optic ray, this introduces a correlation between the residual linear polarization and the position in the beam spot.

For a well-aligned Pockels cell, the leading-order effect will be a spot shape asymmetry. However, a slight misalignment of the Pockels cell breaks the first-moment symmetry of this effect, and the beam divergence and misalignment conspire to create $A_{Q}$ and position differences. While the Pockels cell is typically aligned to the beam within a few milliradians, tests at Jefferson Lab reveal a sensitivity to this effect of $\sim 500 \mathrm{~nm} / \mathrm{mrad}$. Uncorrected, this effect would create unacceptable position differences. Assuming other sources of position differences are under control, the Pockels cell alignment can be tuned to zero measured position differences. Such a procedure uses the divergence effect to counteract contributions from other sources of HCBA.

\subsection{Other effects}

As described in the above section, residual linear polarization and the analyzing power of the photocathode combine to create the majority of HCBA in a well-tuned source. Additional examples of effects seen to be important at Jefferson Lab, other than the various sources of residual linear polarization, are described below.

The Pockels cell is piezo-electric as well as electrooptic, and studies have shown that it deflects the beam as if it were a lens with a helicity-correlated focal length. In practice, carefully centering the beam spot on the crystal minimizes this effect.

A detail of the Jefferson Lab source not shown in fig. 1 is that the laser reflects from several mirrors between the last polarizing element and the Pockels cell. These additional optical elements can degrade the initial polarization state, creating a gradient 
in the linear polarization of the incident beam and therefore in the residual linear polarization at the cathode.

Table 1. Average beam asymmetries under polarization reversal in intensity and energy and beam differences in horizontal and vertical position and angle for G0 (2004) [4] and the HAPPEXII experiments (2005).

\begin{tabular}{|l|c|c|c|}
\hline & G0 & HAPPEX-He & HAPPEX-II \\
\hline $\mathrm{A}_{Q}$ & $-0.28 \mathrm{ppm}$ & $-0.38 \mathrm{ppm}$ & $0.41 \mathrm{ppm}$ \\
\hline $\mathrm{A}_{E}$ & $19 \mathrm{ppb}$ & $3 \mathrm{ppb}$ & $0.2 \mathrm{ppb}$ \\
\hline$\Delta \mathrm{x}$ & $6 \mathrm{~nm}$ & $-0.2 \mathrm{~nm}$ & $0.5 \mathrm{~nm}$ \\
\hline$\Delta \mathrm{x}^{\prime}$ & $2 \mathrm{nrad}$ & $4.4 \mathrm{nrad}$ & $-0.2 \mathrm{nrad}$ \\
\hline$\Delta \mathrm{y}$ & $8 \mathrm{~nm}$ & $-26 \mathrm{~nm}$ & $1.7 \mathrm{~nm}$ \\
\hline$\Delta \mathrm{y}^{\prime}$ & $3 \mathrm{nrad}$ & $-4.4 \mathrm{nrad}$ & $0.2 \mathrm{nrad}$ \\
\hline
\end{tabular}

In general, great care is taken to electrically isolate the helicity signal which triggers voltage changes on the Pockels cell or feedback systems. During the 2005 HAPPEX-He measurement, this signal was not well isolated; leakage of the helicity signal was sufficient to induce large position differences in the $100 \mathrm{keV}$ injector. The DAQ electronics were not affected. This large effect dominated other sources of position differences for this run.

There is cross-talk between the helicity-correlated properties of the three independent beams which run simultaneously for delivery to three separate experimental halls. The physical mechanism of this beam cross-talk at the photocathode is not understood, but has proved to be significant at Jefferson Lab. The experiments which have achieved a very high level of control of position differences each ran as the only high-current beam.

Apertures in the injector are seen to clip the beam in a helicity-correlated manner. In most cases, this effect can be reduced with careful configuration of the beam transport. $A_{Q}$ is monitored throughout the injector to guide the beam optics configuration.

\section{Techniques for reducing HCBA}

Recent experiments at Jefferson Lab have successfully controlled position differences at a high level. In 2004, the G0 experiment used helicity-correlated feedback in the laser source optics to meet goals on intensity and position HCBA. In 2005, improved results were achieved by the HAPPEX Collaboration with relatively small feedback corrections on intensity asymmetry and no feedback loop acting on position differences. A careful configuration of the source laser optics and electron beam transport optics was sufficient for the control of position differences, averaged over the run, at the level of $1 \mathrm{~nm}$. Results for those experiments, including results for the 2005 HAPPEX-He run in which leakage of the real-time helicity-signal drove large beam-orbit deflections, are shown in table 1.

\subsection{Controlling the "PITA" effect}

As described in sect. 2.2, the helicity-correlated difference in the residual linear polarization (arising from the $\Delta$ phase-shift) couples with the analyzing power of the photocathode to create a charge asymmetry $A_{Q}$. This dominant source of $A_{Q}$ can be 
simply tuned out using an asymmetric offset to the voltages applied to the Pockels cell for each helicity state. In fact, a residual linear polarization can be added to the beam to cancel contributions from other birefringent elements, such as the vacuum window. In practice, this global correction is easy to apply: $A_{Q}$ is measured over a range of asymmetry voltage offsets ("PITA voltage") to determine the slope ( $\mathrm{A}_{Q}$ vs. PITA voltage), then the PITA voltage is chosen to zero $A_{Q}$. This correction results in a minimization of asymmetric linear polarization along the cathode analyzing-power axis.

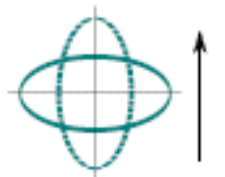

a)

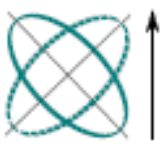

b)

Fig. 4. Illustration of the effect of rotating the polarization ellipses. (a) Aligning the ellipses with the photocathode analyzing power maximizes $A_{Q}$, while (b) an angle of $45^{\circ}$ with respect to the analyzing power axis minimizes $\mathrm{A}_{Q}$.

An independent way of controlling $\mathrm{A}_{Q}$ is by rotating the residual linear polarization to be 45。 to the photocathode analyzing-power axis, as illustrated in fig. 4 . This is accomplished by rotating a half-wave plate (the RHWP), which is located after the Pockels cell as shown in fig. 1. In practice, this wave plate is oriented to provide a small, but non-zero, sensitivity to the cathode analyzing power; this allows a lever arm for a moderate PITA voltage offset to cancel the residual linear polarization induced by other components, such as the vacuum window, while still reducing sensitivity to imperfections in the Pockels cell birefringence.

Using the RHWP to leave only a small sensitivity to the Pockels cell birefringence similarly reduces position differences induced by birefringence gradients of the Pockels cell. The sensitivity is tuned to be non-zero so that the average residual linear polarization due to vacuum window birefringence can be tuned out using the PITA voltage; doing so zeroes the effect of analyzing-power gradients. In this way, these dominant sources of position differences are both highly suppressed.

One significant source which cannot be controlled using the above prescription is the birefringence gradient of the vacuum window. A new polarized gun to be installed at Jefferson Lab will allow for the rotation of the photocathode. When this is installed, the axis of stress-induced birefringence in the vacuum window will be the only arbitrary axis remaining among dominant contributors to position differences. A rotating photocathode analyzing-power axis can be used to zero effects from the vacuum window. This would eliminate the last-known major source of PITA-induced position differences which is presently uncontrolled.

\subsection{Reversals}

Suppression of HCBA may also be provided by slow reversals, in which the sign of the electron beam helicity is reversed relative to the sign of all electronic signals of the helicity (including the Pockels cell voltage). One obvious method for such a reversal would be a Wien polarization filter; however, in practice such devices typically lack sufficient dynamic range. Another method for accomplishing a similar goal is the use of 
an insertable half-wave plate (IHWP) in the source optics, just upstream of the Pockels cell as shown in fig. 1. By reversing the sense of incident linear polarization, this wave plate changes the sign of the phase-shift induced by the Pockels cell and therefore the helicity of the electron beam. This reversal provides an excellent method for canceling asymmetries rooted in electronics pickup of the helicity signal.

However, not all HCBA will cancel under IHWP reversal. In particular, any effect directly related to polarization of the laser light, including all PITA-type effects, change sign along with the polarization and therefore do not cancel. On the other hand, steering/lensing does cancel since this effect is related only to the Pockels cell voltage and not to the polarization of the laser light.

Another type of reversal, which was successfully employed for the SLAC E-158 experiment, is a change of beam energy to create a spin reversal using the $\mathrm{g}-2$ precession. The $\mathrm{g}-2$ reversal is entirely independent of the electronic helicity signal or dynamics of the polarized source. While this reversal is too inefficient to be done frequently and therefore any cancellation will be reduced by slow drifts, it nonetheless remains a full reversal of a large class of effects and is a valuable alternative diagnostic for the integrity of the asymmetry measurement.

\subsection{Feedback systems}

The use of helicity-correlated correctors in the source optics has become standard for the control of $\mathrm{A}_{Q}$ and position differences. While such feedback systems play an important role, they can mask the underlying problems. As one example, consider the Intensity Attenuator (IA) system often used for feedback on $A_{Q}$. The IA consists of a Pockels cell between two linear polarizers, configured so that it can be used as a variable electro-optic shutter. Using such a system, $\mathrm{A}_{Q}$ can be easily controlled, but if large intensity corrections are needed, then underlying problems are only being masked. For example, large $A_{Q}$ would imply a large average $\Delta$ phase shift which would also create large position differences due to analyzing-power gradients. Another correction mechanism for $A_{Q}$ is the PITA voltage set point described above. Such a mechanism may be preferred as it is directly related to the most likely underlying cause of charge asymmetry. In any case, a reasonable guiding principle would be to use feedback only for fine control, after first optimizing the system to reduce the underlying sources of HCBA. Feedback systems for position differences are more problematic. Typically, the laser position is modulated using a piezo-electric actuated mirror or prism. Of course, the general principle still applies: this tool, which does nothing to control the root causes of HCBA, should not be used to hide larger problems. A mirror was successfully used for the $\mathrm{G} 0$ experiment in 2004. For reasons which have not been fully determined, neither the IA nor the piezo-electric actuated mirrors operated on charge or position alone; each device changed both charge asymmetry and position differences. Periodic recalibration of the response matrix of the mirror was required, as well as occasional adjustments to the electron beam transport optics to create a non-singular response matrix [5]. Position feedback might also be performed on the electron beam using magnets, which would remove complicating effects from the photocathode. Such a system has recently been implemented in the injector at Jefferson Lab. 


\subsection{Adiabatic damping}

The problem of helicity-correlated position differences can be reduced by maximizing the benefit of the reduction of beam emittance during acceleration. The $x / x^{\prime}$ phase space is reduced by a factor of $\mathrm{p} 0 / \mathrm{p}$ as the beam is adiabatically accelerated from momentum $\mathrm{p} 0$ to $p$, which implies that helicity-correlated differences in position and angle should each be reduced by a factor of $\sqrt{p_{0} / p}$. For acceleration from the $100 \mathrm{keV}$ injector $(\mathrm{p}=355$ $\mathrm{keV}$ ) to $3 \mathrm{GeV}$ in the experimental Halls, one expects position differences to be reduced by a factor of $\sim 95$.

It is difficult to realize the full benefit of this phase-space reduction for position differences. In transport of the electron beam, correlations develop between $x, x^{\prime}, y$, and $y^{\prime}$ so that the phase-space becomes a rotated ellipsoid. In such a case, while the total phase-space volume remains small, the projection of the phase-space into any coordinate can still be large, so this phase-space coupling still allows helicity-correlated changes in beam position and angle to be large.

While the accelerator optics were originally designed to keep such couplings small, in practice the transport optics are often far from design specifications due to small deviations from specification of beam-line elements or initial conditions. The coupling develops from large-amplitude oscillations around the central trajectory, exposing the beam to aberrations or increased skew contributions from both magnetic and RF acceleration elements. The best strategy for avoiding problematic phase-space coupling is to tune the transport optics back to the original machine design, a process referred to at Jefferson Lab as "matching" the optics. Such matching assures a controlled beam envelope and reduced coupling, and thus maximizes the benefits of adiabatic damping for HCBA.

Major work was invested towards an algorithmic approach to matching transport optics to design specification $[6,7]$. Important diagnostic tools were developed, including a tool for measuring differential orbit launched by a $30 \mathrm{~Hz}$ oscillation of a piezo-electric actuated mirror in the source optics. For the HAPPEX-II hydrogen run in 2005, the transport optics were well matched from the $5 \mathrm{MeV}$ region of the injector through the high-energy region. The expected emittance reduction was verified to within a few percent. The fact that HCBA were very small complicates estimates of the adiabatic damping effect on beam asymmetries, but the reduction in position difference was at least a factor of 5 and, in some estimates, may have been as large as a factor of 30 .

Significant benefits may also be realized from further improvements, in particular, improved control of coupling from the region of the injector before any RF acceleration, where the beam energy is only $100 \mathrm{keV}$. Progress here will be challenging; very small fringe fields can have a large effect on the properties of such a soft beam, and the present configuration of correctors and beam monitors in the region does not support a clean determination of beam parameters.

\section{Conclusions}

Recent progress on controlling HCBA has allowed sub-ppm control of charge asymmetry and $\sim 1 \mathrm{~nm}$ control of position differences. The design and alignment of the polarizedsource laser optics is now guided by an improved understanding of the underlying 
causes of HCBA, and careful configuration of the electron-beam transport optics has been demonstrated to provide significant benefits in the reduction of HCBA. While work in these areas must continue in order to meet the demanding specifications of the nextgeneration experiments, particularly with respect to beam spot size asymmetries, this recent success suggests that these future challenges can be met.

This report details work by members of the HAPPEX Collaboration, the Electron Gun Group, and the Center for Advanced Study of Accelerators at Jefferson Lab. I must specifically acknowledge contributions from G.D. Cates, Y-C. Chao, T.B. Humensky, L.J. Kaufman, and R. Snyder, although many others have also contributed significantly to these efforts. This work was supported by the Department of Energy and by The Southeastern Universities Research Association, Inc. under U.S. DOE Contract No. DEAC05-84150.

\section{References}

1. G.D. Cates et al., Nucl. Instrum. Methods Phys. Res. A 278, 293 (1989).

2. T.B. Humensky et al., Nucl. Instrum. Methods Phys. Res. A 521, 261 (2004).

3. G.D. Cates, Eur. Phys. J. A 24, s2, 109 (2005).

4. K. Nakahara, Eur. Phys. J. A 24, s2, 119 (2005).

5. D.H. Beck, M.L. Pitt, Eur. Phys. J. A 24, s2, 115 (2005).

6. Y.-C. Chao, Proceedings of the Particle Accelerator Conference 2003, JLAB-ACP-0397, http://accelconf.web.cern.ch/AccelConf/p03/PAPERS/TOPB011.PDF.

7. Y.-C. Chao et al., Proceedings of the European Particle Accelerator Conference 2004, JLAB-ACP-04-252 and EPAC-2004-TUPLT163, http://accelconf.web.cern.ch/AccelConf/e04/PAPERS/TUPLT163.PDF. 